\title{
An implant periapical lesion leading to acute osteomyelitis with isolation of Staphylococcus aureus
}

- Shows the virulence of a bacterial species in a normal individual.

- Indicates the significance of replacement of long standing dentures.

- Details the manner in which an implant periapical lesion may occur.

- Highlights the need for appropriate management of implant periapical lesions.

\author{
S. Rokadiya ${ }^{1}$ and N. J. Malden ${ }^{2}$
}

A case of an implant periapical lesion (IPL) proceeding to acute osteomyelitis is presented, most likely due to surface contamination of the implant. Five weeks post placement of two anterior mandibular implants, symptoms of acute pain from one implant presented. This symptom and later swelling were unresponsive to antibiotics. On removal of the implant, there was a purulent discharge which, following microbial analysis, proved to be a pure growth of Staphylococcus aureus. A replacement implant was positioned in the site of the previously lost implant ten weeks later, with no recurrence of infection. Staphylococcus aureus can be isolated commonly from the mouths of denture wearers. When an IPL affects a recently placed implant its removal should be accepted but its replacement also considered.

\section{INTRODUCTION}

Staphylococcus aureus (S. aureus) is the predominant causative organism in long bone osteomyelitis while mandibular osteomyelitis is usually considered a polymicrobial disease. ${ }^{1}$ It is well established that Staphylococci spp may contribute to the development of peri-implantitis although a disease of different aetiology - with a 69\% prevalence of Staphylococci $s p p$ at failing dental implants and a third yielding $S$. aureus. ${ }^{2}$ Recovery of $S$. aureus from IPLs is rare with one reported case from an obvious source. ${ }^{3}$ In addition, retrieval of $S$. aureus from dento-alveolar abscesses and one case of localised alveolar osteitis has been reported. ${ }^{4}$ A case report is presented of an IPL, the successful management of which included the removal and subsequent replacement of an implant.

${ }^{1 *}$ Medical School Undergraduate Office, University of Glasgow Medical School, University Avenue, Glasgow, G12 800; ${ }^{2}$ Oral Surgery Department, Edinburgh Dental Institute, Lauriston Building, Lauriston Place, Edinburgh EH3 9YW

${ }^{*}$ Correspondence to: Dr Suhail Rokadiya

Email: suhail.rokadiya@gmail.com

\section{Refereed Paper}

Accepted 11 July 2008

DOI: 10.1038/sj.bdj.2008.935

${ }^{\circledR}$ British Dental Journal 2008; 205: 489-491

\section{CASE REPORT}

A 73-year-old Caucasian female was referred for the provision of dental implants to aid retention of full lower dentures. The patient had worn the same lower denture for 20 years and replacement dentures were not accepted by the patient. The patient had been a lifelong non-smoker, was otherwise healthy and was taking no medication. Following examination and radiographic assessment an atrophic Class $\mathrm{V}$ ridge was diagnosed in the mandible, and a decision was made to place implants in the canine regions in accordance with the McGill consensus statement on overdentures. ${ }^{5}$ Treatment was provided under intravenous sedation. While adhering to standard cross infection control measures, one Straumann titanium implant was placed in both lower canine regions following the exposure of bone via the minimal raising of separate mucoperiosteal flaps. Good initial mechanical stability was noted. Post-operative instructions, analgesia and chlorhexidine $0.2 \%$ mouth rinse were provided for daily use.

At review, six days post-operatively, the patient was pleased with the outcome, and oral hygiene was considered to be good. A postoperative OPG was obtained; left implant area

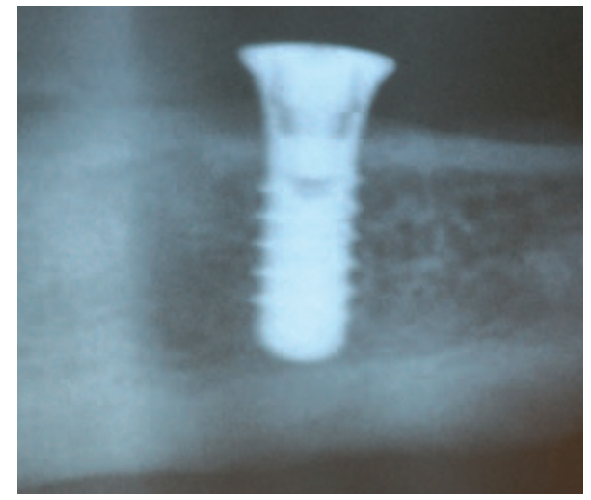

Fig. 1 Six days post placement, good mechanical retention, no symptoms

detail is shown in Figure 1. Ten days post-operatively one of the cover screws was lost and replaced the same day. No further problems were reported by the patient. However, seven days later (17 days post-operatively), the patient was seen at a local district general hospital complaining of an acute gnawing pain in the chin on the left side. She was prescribed penicillin and while the symptoms responded initially, they returned with increasing intensity the following fortnight at which point she presented to the Oral Surgery Department. The pain was again described as a gnawing abscess-like pain which occasionally throbbed and radiated to the lip on the left side. This did not respond to antimicrobial therapy. A further left side 
panoramic view was obtained which revealed a radiolucency apical and distal to the left implant indicating an IPL (Fig. 2). No communication was detected between the mucosa and apex of the implant, indeed the implant was considered to be firmly retained within the bone. The following day, the painful symptoms were worsening and a bilateral swelling of the lower lip was obvious. The implant was then removed, upon which there was a purulent discharge of creamy pus and consequent immediate reduction in pain. A diagnosis of acute osteomyelitis was made and flucloxacillin was empirically prescribed. Bacteriological identification of the pus revealed a yield of $S$. aureus.

On review two weeks subsequently, it transpired the patient had not taken the flucloxacillin due to the reduction in pain and resolution of her lip swelling almost immediately following implant removal. Eight weeks later a further implant was placed in the previously removed implant site. This was successfully used as an overdenture retainer four months later.

\section{DISCUSSION}

A previous report of osteomyelitis ${ }^{3}$ subsequent to implant therapy stemmed from placement of a titanium implant into an immediate extraction socket to which infection tracked from an adjacent tooth. Retained root tips have also been cited as a nidus of infection. ${ }^{6,7}$

In an IPL the source of infection is most frequently associated with endodontic pathology affecting an adjacent tooth. ${ }^{6}$ Haematogenous infection ${ }^{8}$ has also been referred to in relation to early failure of an implant, although there are growing doubts concerning this mode of spread. ${ }^{9}$ Haematogenous seeded osteomyelitis in the long bone metaphyseal plate is recognised in the elderly patient. ${ }^{1}$

IPLs are rare, with a prevalence of $0.26 \%{ }^{10}$ usually in dense bone ${ }^{7}$ such as the mandible. Characteristically periapical bone rarefaction is a sign of early failure ${ }^{7}$ and there may be no evidence of bacterial infection. ${ }^{11}$ Although there was no indication of this on the pre-operative OPG radiograph, in our patients' case no intra-oral films were taken which may have allowed greater acuity as periapical radiographs detect approximately $40 \%$ more osseous defects that OPGs. ${ }^{12}$ In practice, however, this is difficult to achieve in the edentulous patient.

The symptoms must be immediately recognised, radiologically assessed and rapidly surgically managed to decrease tissue damage ${ }^{6}$ and pain. If mobile, the implant should be considered to have failed and be immediately removed. ${ }^{10}$ Treatment may include meticulous debridement for removal of the infectious source, ${ }^{13}$ although complete debridement is technically difficult and may necessitate removal of the implant. ${ }^{6}$ If debridement is attained, it may be followed by application of a bacteriostatic agent. ${ }^{9}$ Treatment should be carried out within one month of IPL onset, ${ }^{6}$ although small inactive (non-infective) lesions can be monitored. ${ }^{10}$ It is likely that in our patient's case the virulence of the bacteria involved meant there was a rapid advancement to osteomyelitis within two weeks of implant provision and with minimal signs of failure prior to this.

There are many possible sources of bacterial contamination during implant surgery including the surgical instruments, gloves, air in the operating room, air expired by the patient, saliva in the oral cavity, or the peri-oral skin.,8 Although there is a possibility of poor surgical technique being the cause of infection in this case, we believe that there was standard and meticulous aseptic technique used. We propose that the isolation of colonies of $S$. aureus from the infected wound site indicates the infection was likely due to commensal pathogens. Indeed analysis with oral swabs, aspirates and oral rinses ${ }^{4}$ show that elderly patients may more frequently harbour $S$. aureus in their mouths than previously suspected..$^{14}$ Frequency of isolation of Staphylococcus spp may be age related ${ }^{15}$ and it is regularly recovered from denture plaque. ${ }^{16} \mathrm{~S}$. aureus colonises denture surfaces and is not eliminated with standard denture cleansers. ${ }^{4}$ Rinsing with chlorhexidine has been found to decrease infective complications during the implant submerged period, ${ }^{17}$ however, $S$. aureus may have a particular avidity to titanium surfaces. ${ }^{18}$ Immunological factors may

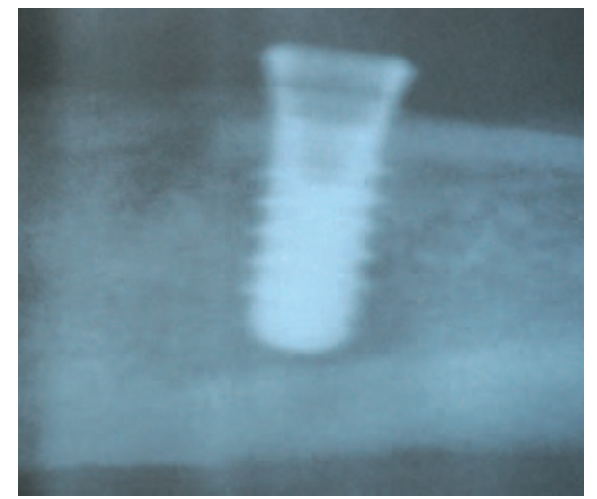

Fig. 2 Twenty-eight days post placement, radiolucency indicating osteolysis apicodistally to the implant which was still showing good mechanical retention

also be involved.$^{19}$ The surgeon's utmost care in not infecting the implant prior to implantation is paramount and the chance of contamination by the saliva can be reduced. This can be augmented by methods such as decreasing salivary flow with atropine, ensuring the patient is in a supine position, protection of the surgical site by orally pedunculated flaps and using separate aspiration tips for saliva and the surgical site ${ }^{20}$ may be employed. Research is required into the likelihood of oral commensal bacteria causing infection in subsequent cases such as this. Colonies may be typed and oral commensal cultured and matched. There may be a case for the re-making of existing old dentures in patients prior to implant surgery in order to decrease the salivary concentration of $S$. aureus.

1. Scolozzi P, Lombardi T, Edney T, Jaques B. Enteric bacteria mandibular osteomyelitis. Oral Surg Oral Med Oral Pathol Oral Radiol Endod 2005; 99: e42-46.

2. Rams T E, Feik D, Slots J. Staphylococci in human periodontal diseases. Oral Microbiol Immunol 1990; 5: 29-32.

3. Sussman H I, Moss S S. Localized osteomyelitis secondary to endodontic-implant pathosis: a case report. J Periodontol 1993; 64: 306-310.

4. Smith A J, Robertson D, Tang M K, Jackson M S et al. Staphylococcus aureus in the oral cavity: a three-year retrospective analysis of clinical laboratory data. Br Dent J 2003; 195: 701-703.

5. Feine J S, Carlsson G E, Awad M A, Chehade A et al. The McGill consensus statement on overdentures. Mandibular two-implant overdentures as first choice standard of care for edentulous patients. Montreal, Quebec, May 24-25, 2002. Int J Oral Maxillofac Implants 2002; 17: 601-602.

6. Park S H, Sorensen W P, Wang H L. Management and prevention of retrograde peri-implant infection from retained root tips: two case reports. Int J Periodontics Restorative Dent 2004; 24: 422-433.

7. Esposito M, Hirsch J, Lekholm U, Thomsen P. Differential diagnosis and treatment strategies for biologic complications and failing oral implants: a review of the literature. Int J Oral Maxillofac Implants 1999; 14: 473-490. 
8. Quirynen M, Teughels W. Microbiologically compromised patients and impact on oral implants. Periodontol 2000 2003; 33: 119-128.

9. Flanagan D. Apical (retrograde) peri-implantitis: a case report of an active lesion. J Oral Implantol 2002; 28: 92-96.

10. Reiser G M, Nevins M. The implant periapical lesion: etiology, prevention, and treatment. Compend Contin Educ Dent 1995; 16: 768, 770, 772.

11. Scarano A, Di Domizio P, Petrone G, lezzi G Piattelli A. Implant periapical lesion: a clinical and histologic case report. J Oral Implantol 2000; 26: 109-113

12. Pepelassi E A, Tsiklakis K, Diamanti-Kipioti A. Radiographic detection and assessment of the periodontal endosseous defects. J Clin Periodontol
2000; 27: 224-230.

13. Oh T J, Yoon J, Wang H L. Management of the implant periapical lesion: a case report. Implant Dent 2003; 12: 41-46.

14. Bagg J, Sweeney M P, Harvey-Wood K, Wiggins A Possible role of Staphylococcus aureus in severe oral mucositis among elderly dehydrated patients. Microbiol Ecol Health Dis 1995; 8: 51-56.

15. Percival R S, Challacombe S J, Marsh P D. Agerelated microbiological changes in the salivary and plaque microflora of healthy adults. J Med Microbiol 1991; 35: 5-11.

16. Marsh P D, Percival R S, Challacombe S J. The influence of denture-wearing and age on the oral microflora. J Dent Res 1992; 71: 1374-1381.

17. Lambert P M, Morris H F, Ochi S. The influence of
$0.12 \%$ chlorhexidine digluconate rinses on the incidence of infectious complications and implant success. J Oral Maxillofac Surg 1997;

55(12 Suppl 5): 25-30.

18. Karlov A V, Khlusov I A, Pontak V A, Ignatov V P et al. Adhesion of Staphylococcus aureus to implants with different physicochemical characteristics. Bull Exp Biol Med 2002; 134: 277-280.

19. Kronström M, Svenson B, Hellman M, Persson G R. Early implant failures in patients treated with Brånemark System titanium dental implants: a retrospective study. Int J Oral Maxillofac Implants 2001; 16: 201-207.

20. Quirynen M, De Soete M, van Steenberghe D. Infectious risks for oral implants: a review of the literature. Clin Oral Implants Res 2002; 13: 1-19. 